\title{
Experimental Research on the Behaviour of Heat Pipe Using Graphene Oxide as Working Fluid
}

\author{
Muthukumar M, Senthilkumar R, Vaidyanathan S
}

\begin{abstract}
The liberation of heat from the electronic devices used in the industries, refrigeration and etc. is main concern today. The temperature of the planet earth is being increasing day by day, the generation of heat by the manufacturing industries should be reduced using innovative techniques. Generally a heat exchangers were used to reduce it. In this study, graphene oxide employed as as working fluid to determine the effective thermal conductivity. The working fluid graphene oxide plays vital role in dissipation of heat from the evaporation zone to condensed zone. The experiment setup was done with about $19.1 \mathrm{~mm}$ and $21 \mathrm{~mm}$ inner and outer diameter, respectively. The length of the heat pipe is $600 \mathrm{~mm}$. The wick material was made up of brass. We observed that the graphene oxide improved thew efficieny by using as working fluid.
\end{abstract}

Keywords: Graphene oxide, Heat pipe, Working fluid, Thermal conductivity.

\section{INTRODUCTION}

Heat pipe is a device used for heating and cooling which can transport excessive amount of warmth from one place to next place with small temperature difference through small cross sectional area by high thermal conductance. It is a powerful passive device employed to carry the heat. It prevents the heat transfer equipment and other devices from the possible of overheating. The heat pipe is a perfect instrument for the heat control of electronic components. It exhibits highly heat transfer characteristics, like the capacity to transfer more volume of heat from one point to another point under various heat loads. There are different types heat transfer devices available to minimize the heat generation in the equipment. The heat pipe, a strong system among other modern-day appliances, favoured transferring the big quantity of heat without external sources [1]. Heat pipe as a two phases of heat transfer instrument is generally called as super conductor of heat, has been employed for cooling of high power electronic devices, because it has elevated heat conductivity. Due to highly flexible of heat pipes, the achieving and controlling the thermal gradient. It has some characteristic features like controlling ability, plan and assembling simplicity, little start to finish temperature drops. With this in mind, in this study, graphene oxide was employed as working fluid to reduce heat production in pipes.

Revised Version Manuscript Received on 10 September, 2019.

Muthukumar M, Research Scholar, Department of Mechanical Engineering, Annamalai University, Chennai, Tamil Nadu, India.(email: thavasi26@gmail.com)

Senthilkumar R, Assistant Professor, Department of Mechanical rsklme@yahoo.com) Annamalai University, Tamil Nadu, India.(email: yesvee_123@rediffmail.com) Engineering, Annamalai University, Tamil Nadu, India.(email:

Vaidyanathan S, Professor, Department of Mechanical Engineering,

\section{DRAWBACKS IN HEAT TRANSFER}

The following parameters can be considered for the behaviour of the warmth pipe and tasks are the container shape, working liquid and wick structure. Depending upon the applications, a few heat pipes are designed to give less warmth load to more heat loads. When the heat pipe working beyond its limits leading to reduce thermal conductivity. It became necessary to ensure the heat pipe and its heat load safely. At steady conditions the capacity of heat pipe to carry that heat is regulated by several limitations. The limitations are because to sonic, narrow, bubbling, entrainment, and continuum vapour, vapour pressure frozen begins up and condenser impacts. The limitation can be noticed in related to size and the state of the pipe, the working liquid, wick structure and working temperature separately [2]. From the above limitations as far as possible, sonic point, boiling entrainment farthest and viscous cutoff plays crucial role in heat pipe operation.

\section{CAPILLARY LIMIT}

For a compelling warmth pipe operation, the hair like pressure developed by this grooved structure is always larger than the total of the pressure misfortunes occurs during liquid stream path. The maximum heat exchange reached in heat pipe is governed under conditions such that the capillary pressure head is greater than or same to the average pressure losses comes with liquid path. Considering this gradual heat pipe work, the pressure balance is satisfied. When the capillary pressure difference is equal to the sum of pressure losses, the capillary structure provides the return of condensate to the evaporator to prevent the dry-out point This condition may differ due to wick structure, working liquid, operating temperature, evaporator warm motion and stream channel. The average pressure loss is greater than the capillary pressure the difference, the condensate do not move here the condenser to evaporator section and it leads the wick structure to dry-out and heat pipe gets overheated. In normal working conditions, as far as limit resolves the utmost heat move rate.

\section{Sonic Limit}

When the velocity of vapour in evaporator section reaches the local speed velocity, the flow is chocked. When chocked condition, the vapour flow will not reach the evaporator related to the working fluid and heat pipe attained the maximum transport is said to be sonic limit. Due to sonic 
limit, there is an increase in pressure drop from the evaporator to condenser.

\section{Boiling Limit}

The boiling limit happens when evaporator thermal flux is sufficient to induce nucleate boiling. It generates vapour bubbles that partly obstructs the liquid returns and it results in wick in the evaporator dry out. The boiling limit is also considered to be the heat flux limit. It is always related to heat pipes of non-metallic working fluids. Usually the boiling limit Is considered as very uncommon in liquid metal heat pipes [3].

\section{Entrainment Limit}

A frictional shear stress occurs at the liquid-vapour interface since liquid and vapour moves in the converse direction may slow down the condensate liquid to the evaporator. The effects induced by the frictional shear stress on the liquid vapour interface, condensate cannot return to the evaporator. At this point, the liquid becomes dry in the evaporator. The heat pipe attains a heat transport at this limit which termed as an entrainment limit.

\section{Viscous Limit}

When the vapour pressure from the evaporator to the condenser cannot overcome the pressure drop caused by the viscous force, the heat pipe attains the heat transport limit and it is called as viscous limit. The vapour pressure in the condenser is equal to zero when the viscous limit is reached. operating temperature. Hence, the alternative working fluid must be adopted in order to increase operating temperature.

\section{GRAPHENE OXIDE}

Graphene is a carbon atom obtained from graphite fabric. it's far a dimensional fabric because the top of the material is considered as zero. One millimeter of graphite contains 3 million layers of graphene. Graphene has materialized as one of the perfect promising nano materials because of its inimitable mixture of fantastic properties consisting of its high surface place (2630 m2g-1), Youngs modulus (1 TPa) and thermal conductivity (5000 Wm-1K-1) [4]. It isn't always most effective one of the thinnest but also strongest substances. It conducts warmth higher than all different substances. it's miles a great conductor of strength. it's miles optically obvious, yet so dense that it's far impermeable to gases, even the helium gasoline cannot penetrate via it. Graphene owns other wonderful features such as high electron mobility is a hundred times faster than silicon, it conducts heat 2 times higher than diamond and it has thirteen times better electrical conductivity than copper.

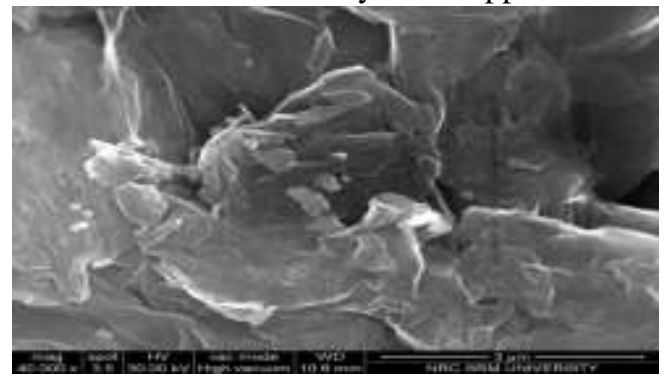

Fig. 1 SEM image of graphene oxide nano particle This causes the heat pipe to function below the suitable

To prepare the nano fluids (Graphene oxide), DI water is utilized as the base fluid. The $100 \mathrm{mg}$ of graphene oxide nanoparticles are dispersed in one litre of DI water. The ultrasonic homogenizer is employed to prepare the nano fluid and prevent the particle agglomeration. The ultrasonic homogenizer is operated for 10 hours in order to vibrate the resulting suspension which results in stable suspension. The prepared solution is employed as working fluid for heat pipe as the nanoparticles are suspended in DI water entirely after $10 \mathrm{hrs}$. The above procedure is repeated for all the nanoparticles to prepare other nano fluids. The ultrasonic homogenizer helps to improve dispersion behavior, uniformity, stability and continuous suspension of nanoparticles in the base fluid. In this research graphene oxide is used as nano fluid. Nano fluid is used as a substitute for water in heat pipes. Nano fluids has a lot of applications in various industries for various purposes such as efficient heat transfer, cooling of electrical devices, refrigeration, thermal storage, lubrication and etc.[5][6].

\section{EXPERIMENT}

The schematic representation of Figures 2 and 3 demonstrates the experimental setup of graphene oxide in heat pipe as working fluid. The specs of heat pipe are tabulated in table 1 . heat input turned into generated close to to evaporator section thru electrical deliver with the help of heater which is sure with right electric insulation. The preferred warmth enter have been furnished at give up of evaporator with the aid of regulating the valve. Water jacket is furnished near to condenser quit which get rid of the heat. It has the capacity to transfer the warmth thru the interior structure. therefore, there may be a scourge of wall temperature happens ends in damage of warmth pipe, if not released at the condenser properly. therefore, earlier than warmness was provided to the evaporator, the cooling water became first disbursed via the condenser jacket. there has been an increase of innovative power enter raised to the preferred degree.

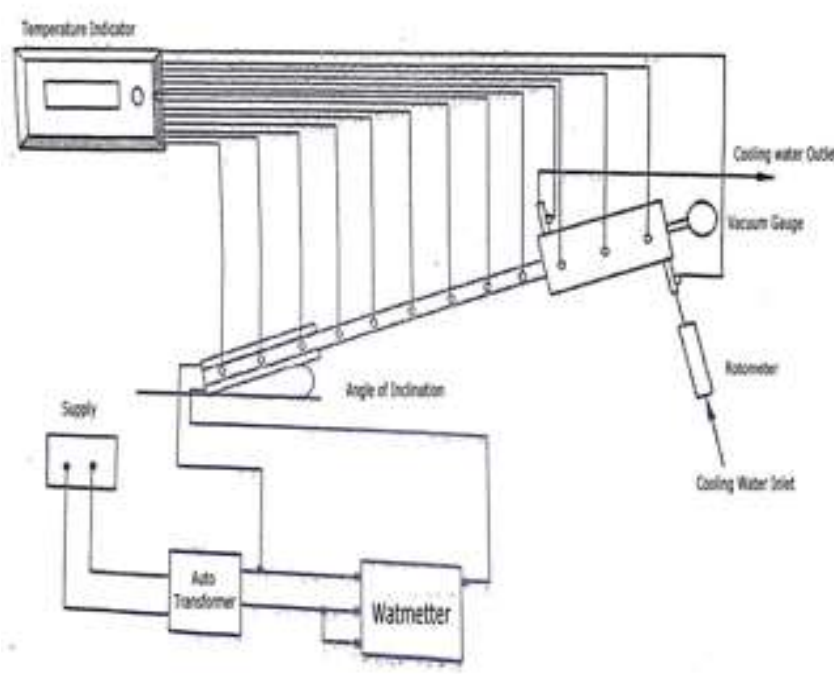

Fig.2 Schematic diagram of experimental setup 
The outside temperatures at 10 extraordinary areas in warm temperature pipe have been predicted the use of $\mathrm{T}$ kind thermocouple at a predictable time interim of 5 minutes till warm temperature pipe stretches regular usa situation. Out of ten, three thermocouples are situated in the evaporator, 3 at the condenser and staying at the evaporator section. numerous thermocouples were applied to control the water gulf and outlet throughout the condenser section. The facts electricity have been killed, when arrives on the steady specific, a cooling water become authorized to route through the condenser to direct the temperature to start the analyses. At that factor the power turned into increased to the following stage and the warm temperature pipe modified into attempted for its presentation. Exploratory strategies have been rehashed for various filling proportions, numerous circulate charges and numerous dispositions of the warmth pipe concerning the extent and perceptions have been recorded.

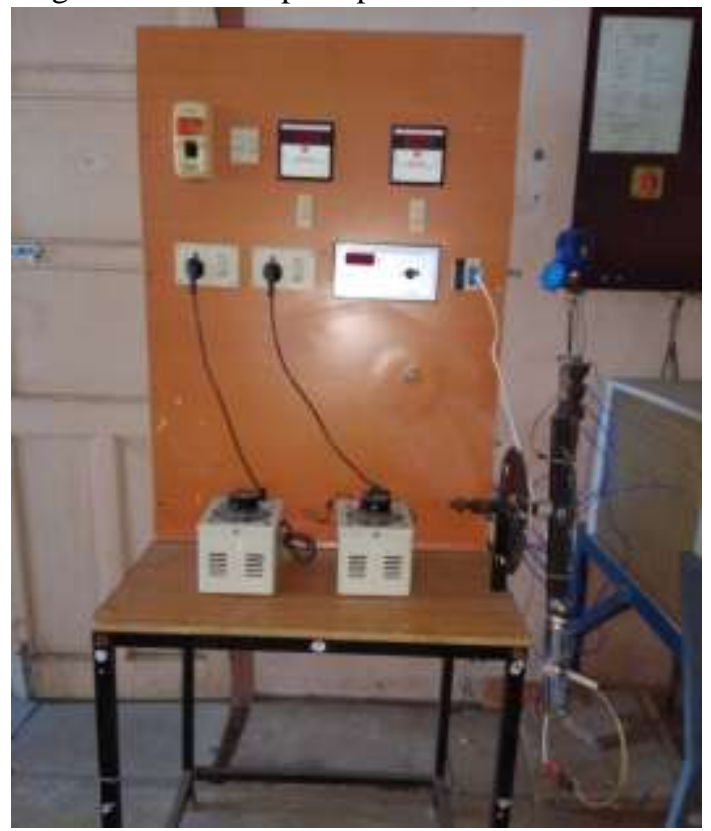

Fig.3 Experimental setup of heat pipe using graphene oxide as working fluid

Table 1. Heat Pipe Specifications

\begin{tabular}{|l|l|}
\hline Specification & Dimensions \\
\hline Outside diameter, $\mathrm{m}$ & 0.021 \\
\hline Inside diameter, $\mathrm{m}$ & 0.0191 \\
\hline Evaporator length, $\mathrm{m}$ & 0.150 \\
\hline Condenser length, $\mathrm{m}$ & 0.150 \\
\hline Adiabatic length, $\mathrm{m}$ & 0.300 \\
\hline Total length, $\mathrm{m}$ & 0.600 \\
\hline Working Fluid & Graphene Oxide \\
\hline Wick mesh size, & 80 per Sq. inch \\
\hline No. of layers of wick & 2 \\
\hline
\end{tabular}

\section{RESULTS AND DISCUSSIONS}

The contribution to the evaporator. An expansion of heat skills is distinctly diagnosed with increasing in warmth transition, firmly recognized with the manner that the temperature trade the various evaporator and condenser increments coming approximately in to higher vanishing of warmth skip tempo of graphene oxide. At better warm temperature contribution to evaporator region, the outside of graphene oxide as running medium is higher and it roots the running medium recognized with vapor factors into condenser section. the nice and cozy effectiveness increments in filling percentage recognized with strolling liquid - graphene oxide within the evaporator as a lot as $80 \%$ of quantity, a short time later it'll in general abatement of drift vapor and fluid gets reduced. because of heat skillability receives dwindled while stacked to graphene oxide surpasses above eighty\%. Figures 4-eight demonstrates the warm effectiveness of graphene oxide as running liquid in wonderful proportions and fringe of tendency.

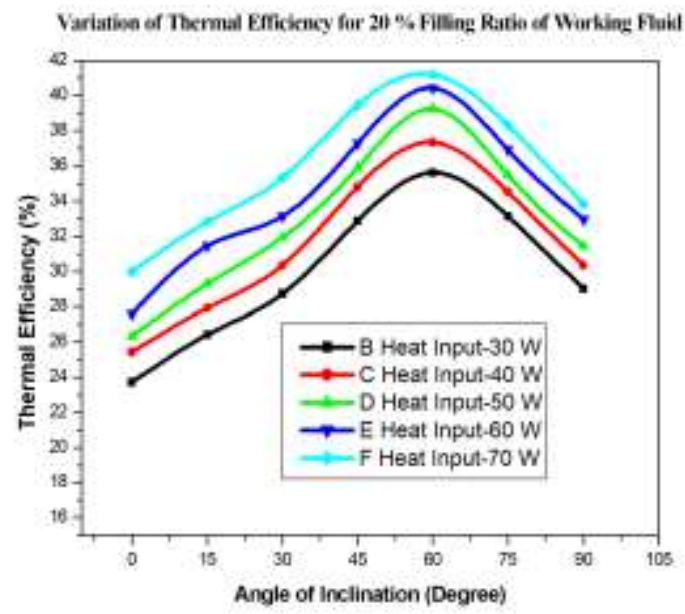

Fig. 4 Thermal efficiency of heat pipe with $20 \%$ of filling ratio of working fluid

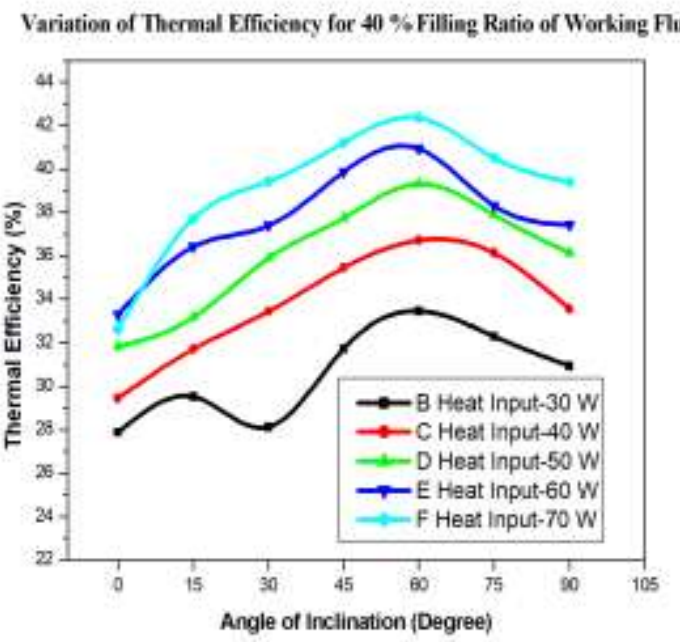

Fig. 5 Thermal efficiency of heat pipe with $40 \%$ of filling ratio of working fluid 


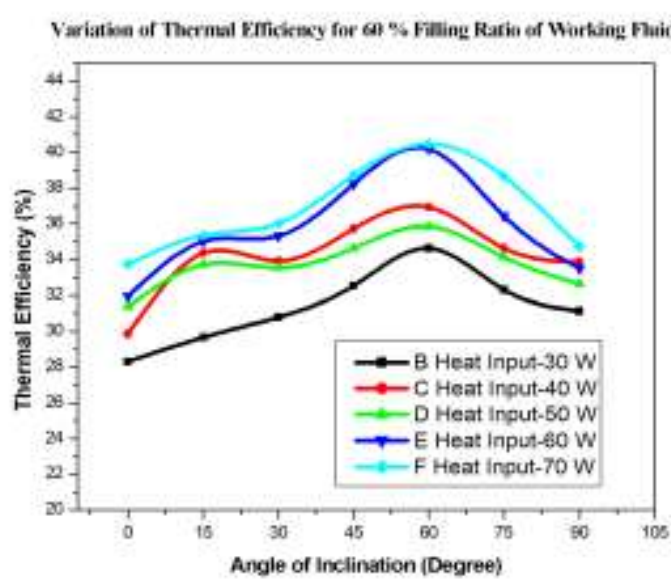

Fig. 6 Thermal efficiency of heat pipe with $60 \%$ of filling ratio of working fluid

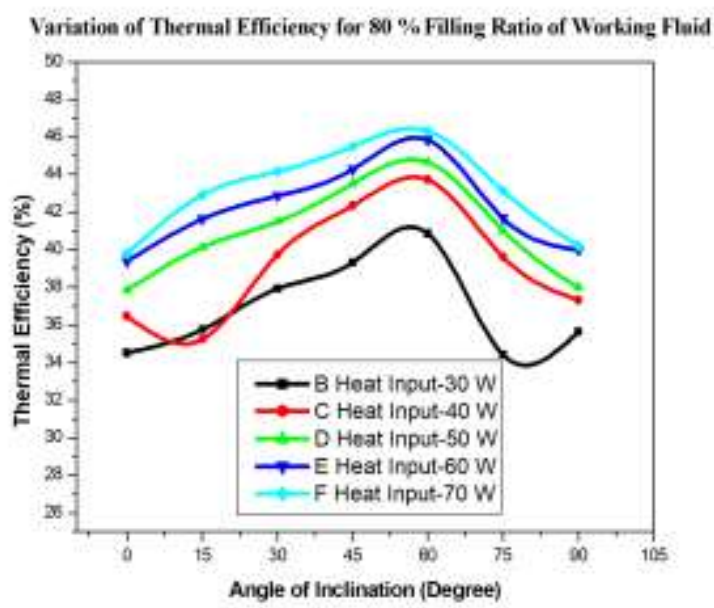

Fig. 7 Thermal efficiency of heat pipe with $80 \%$ of filling ratio of working fluid

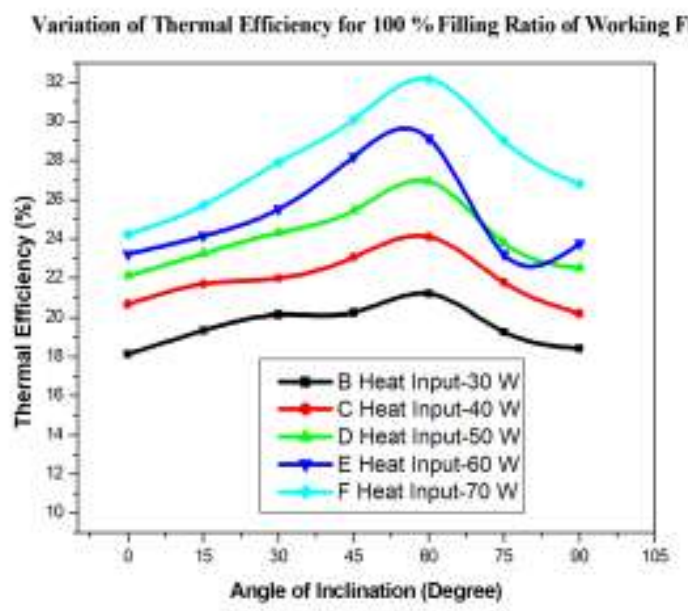

Fig. 8 Thermal efficiency of heat pipe with $100 \%$ of filling ratio of working fluid

From the perceptions it's miles resolved that the first-class and comfortable talent of the warmth pipe with numerous warmth records and various thing of tendency increments with expansion of graphene oxide as working liquid. there is a variety in warm productiveness when the recording percentage of graphenae oxide is increments with $20 \%$, forty $\%, 60 \%, 80 \%$. on the point while the filling proportion of graphene oxide is extended often eighty $\%$, for example $100 \%$ the nice and cozy proficiency receives faded. it's also installation that, the warm productiveness at an edge of tendency of $60^{\circ}$ is higher for all of the warmth channels with numerous filling share of graphene oxide as operating liquid.

\section{CONCLUSION}

The accompanying results are gotten from the test examinations for the graphene oxide as jogging medium. warm talent can be impacted with the aid of some additives such as warmth enter, operating liquid, and fringe of tendency.

1. the threshold of tendency for higher warmness talent is executed at an edge of $60^{\circ}$.

2 . the warm effectiveness with graphene oxide increments with a variety in fringe of tendency from zero- $60^{\circ}$ degree.

three. the warmth effectiveness of heat pipe with graphene oxide diminishes with a thing of tendency more than $60^{\circ}$. as an instance at ninety ${ }^{\circ}$ component of tendency.

4. the heat productivity of heat pipe increments with a ramification of filling percentage of graphene oxide upto $80 \%$.

five. the warm productiveness of heat pipe diminishes with increment of stage of filling percentage more than eighty $\%$ for example $100 \%$.

6 . the heat productivity increments with a ramification of heat input. The warmth contribution of $70 \mathrm{~W}$ offers higher heat effectiveness with diverse filling percentage of graphene oxide and fringe of tendency

\section{REFERENCES}

1. Kyung Mo Kim and In Cheol Bang, "affects of graphene oxide nanofluids on warmth pipe execution and hairlike cutoff elements," global journal of Thermal Sciences, one hundred, pp.346-356, 2016 https://doi.Org/10.1016/j.Ijthermalsci.2015.10.half.

2. Faghri, A., warmth Pipe science and generation, First version, Taylor and Francis., Washington, 1995.

3. Faghri, A.(2014), warmness Pipes: evaluation, openings and problems, Frontier in warmness pipes, Vol.5(1), Feb. pp.1-forty four.

4. Yanwu Zhu et.Al., "Graphene and graphene oxide: Synthesis, houses, and packages," superior materilas, wileyonlinelibrary.Com, Vol.22, pp. 3906 - 3924, 2010 , DOI: 10.1002/adma.201001068.

5. Wei $\mathrm{Yu}$ and Huaqing Xie, "An audit on nanofliuds: coaching, stability systems, and applications," magazine of Nanomaterials, Vol. 2012, pp.1-17, January 2012. Doi:10.1155/2012/435873

6. Mohamoud Jama et.Al. "fundamental audit on nanofluids preparations, Characterization, and programs," journal of Nanomaterials, Vol. 2016, pp. 1-22, January 2016, DOI: $10.1155 / 2016 / 6717624$. 